\title{
Computer aided geometric feasible assembly sequence planning and optimizing
}

\section{Qiang Su}

(C) Springer-Verlag London Limited 2006

Due to an unfortunate error there were mistakes in some of the equations.

1) All the equations of " $I=[0,2 \pi]$ " should be changed to " $I=[0,2 \pi)$ ".
2) The Eq. 16 should be as follows

$$
\begin{aligned}
A A_{A S_{i}} & =\left(\prod_{j=1}^{n}\left(A A_{P_{j}^{i}}\right)^{k_{P_{j}^{i}}}\right)^{\frac{1}{\sum k_{P_{j}^{i}}}} \\
& =\left(\prod_{j=1}^{n}\left(I-\bigcup_{k=1}^{j-1} C D_{P_{j}^{i}, P_{k}^{i}}\right)^{k_{P_{j}^{i}}}\right)^{\frac{1}{\sum k_{P_{j}^{i}}}}
\end{aligned}
$$

The online version of the original article can be found at: http://dx.doi. org/10.1007/s00170-006-0447-0.

Q. Su $(\bowtie)$

Department of Industrial Engineering and Management,

Shanghai Jiaotong University,

1954 Huashan Road,

Shanghai 20030, People's Republic of China

e-mail: suq@sjtu.edu.cn 